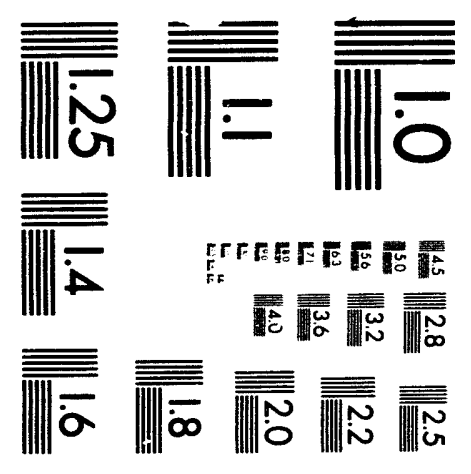



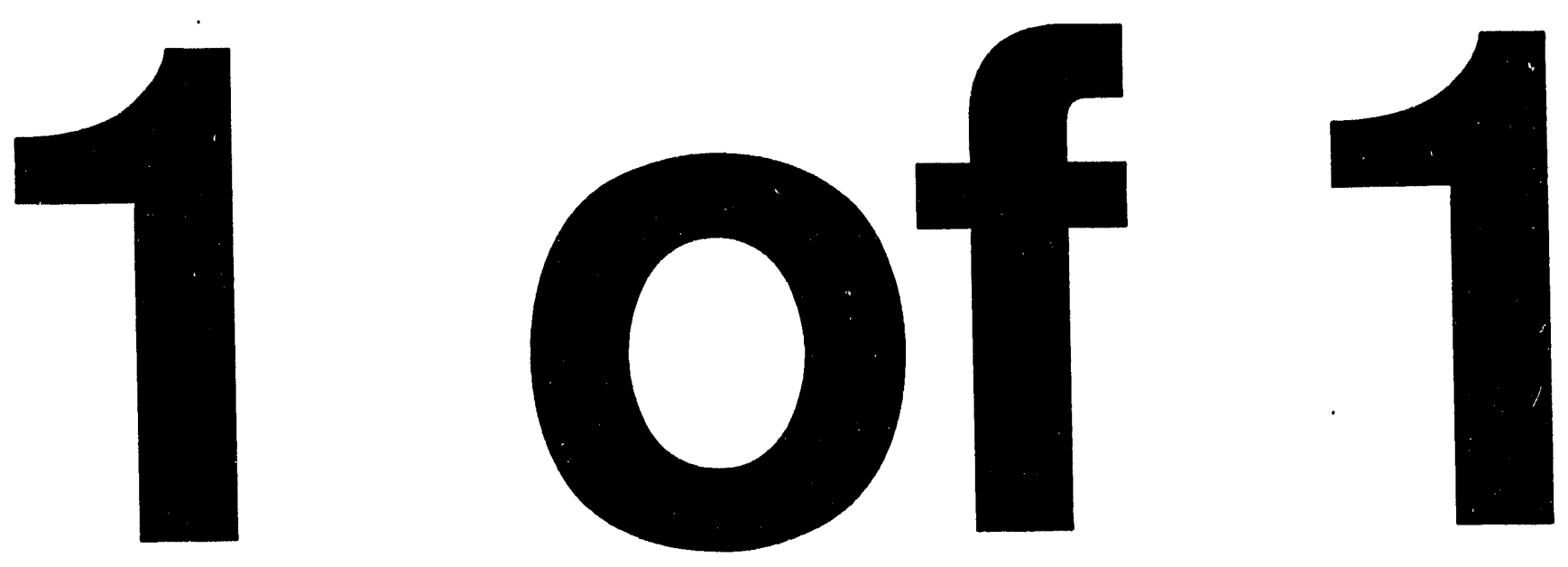


\title{
Matrix elements for the decays of S- and P-wave quarkonium:
} an exploratory study.

\author{
G. T. Bodwin, S. Kim and D. K. Sinclair *
}

a HEP Division, Argonne National Laboratory, 9700 South Cass Avenue, Argonne, Illinois, 60439, USA

Bodwin, Brasten and Lepage have shown that, in a non-relativistic expanaion in powers of the heary-quark velocity, the decay rates of S- and P-wave quarkonia can be written as aums of products of a hard parton matrix element, which can be calculated perturbatively, times a low-momentum expectation value of a parton operator in the quarkonium state. We report on attempts to calculate these low momentum matrix elements on the lattice in the non-relativistic formulation of the quark dynamics. The quenched approximation in used throughout.

The charmonium and $b \bar{b}$ syotems are nonrelativistic in the sense that $v^{2} \ll 1$ where $v$ is the heavy-quark velocity $\left(v^{2} \sim 0.2-0.3\right.$ for charmonium $v^{2} \sim 0.1$ for $\Upsilon$ ). The annihilation of the $Q \bar{Q}$ ( $Q$ is a b or c quark) pair is suppressed, and occurs, when it does, at distances $\sim 1 / M_{Q}$. Bodwin, Braaten and Lepage [1] have shown that these facts allow one to write quarkonium decay rates as a sum of terms, each of which factors into a long-distance part times a short-distance part. The long-distance part involves the distribution of heavy quarks in quarkonium; the shortdistance part can be calculated perturbatively. In particular, the S- and P-wave decay rates can be written at lowest non-trivial order in $v^{2}$ as

$$
\begin{aligned}
& \Gamma\left({ }^{20+1} S_{J} \rightarrow X\right)=G_{1}(S) \hat{\Gamma}_{1}\left(Q Q\left({ }^{20+1} S_{\jmath}\right) \rightarrow X\right) \\
& \Gamma\left({ }^{20+1} P_{J} \rightarrow X\right)=H_{1}(P) \hat{\Gamma}_{1}\left(Q Q\left(^{20+1} P_{J}\right) \rightarrow X\right) \\
& +H_{8}(P) \hat{\Gamma}_{8}\left(Q \bar{Q}\left({ }^{2 s+1} S_{J}\right) \rightarrow X\right),
\end{aligned}
$$

where the $X$ 's represent states of light partons. The $\hat{\Gamma}^{\prime}$ 's are the short-distance $\left(p \sim M_{Q}\right)$ partonlevel decay rates. $G_{1}, H_{1}$ and $H_{8}$ are the longdistance $\left(p \sim M_{Q} v, E \sim M_{Q} v^{2}\right)$ matrix elements that we calculate on the lattice.

In our lattice calculations we have used 149 independent equilibrated quenched gauge configurations on a $16^{3} \times 32$ lattice with $\beta=6.0$. Heavyquark, and hence quarkonium propagators were

Talk presented by D. K. Sinclair

This work was supported by the U. S. Department of Energy, Contract No. W-31-109-ENG-38. calculated using the non-relativistic formulation of Lepage and collaborators [2]. We used the lattice version of the quark action that is based on the euclidean lagrangian

$$
\mathcal{L}_{Q}=\psi^{\dagger}\left(D_{t}-\frac{D^{2}}{2 M_{Q}}\right) \psi+\chi^{\dagger}\left(D_{\imath}-\frac{D^{2}}{2 M_{Q}}\right) \chi_{1}
$$

which is valid to the lowest non-trivial order in $v^{2}$. We calculate the quark Green's function which obeys the evolution equation [2]

$$
\begin{aligned}
G(x, t+1) & =\left(1-H_{0} / 2 n\right)^{n} U_{x, t}^{\dagger}\left(1-H_{0} / 2 n\right)^{n} \\
& \times G(x, t)+\delta_{x, 0} \delta_{t+1,0},
\end{aligned}
$$

with $G(x, t)=0$ for $t<0$, and $H_{0}=$ $-\Delta^{(2)} / 2 M_{0}-E_{\text {sub }}$. Here $\Delta^{(2)}$ is the gaugecovariant discrete laplacian and $M_{0}$ the bare quark mass. $E_{\text {sub }}$ allows one to choose the zeropoint energy.

For these preliminary calculations point sources and sinks were chosen for the quark Green's functions. This enabled us to use sources for the quark Green's functions at the site of the four-fermi operators that appear in $G_{1}, H_{1}$ and $\mathrm{H}_{8}$, making it simple to calculate matrix elements in which the quarkonia are at zero momentum and on arbitrary time-slices, as well as to extract quarkonium wave functions (or their first derivatives) at the origin.

For charmonium we chose $M_{0}=0.5, n=3$ and $E_{\mathrm{dub}}=1.11$, in lattice units. In converting to "physical units" we chose $1 / a=2 \mathrm{GeV}$. In

DSTImBUTION OF THIS DOCURERT IS URLIMITED
MASTER $d \theta$ 


\section{DISCLAIMER}

This repon was prepared as an account of work sponsored by an agency of the United States Government. Neither the United States Government not any agency thereof, nor any of their employees, makes any warranty, express or implied, or assumes any legal liability or responsibility for the accuracy, completeness, or usefulness of any information, apparatus, product, or process disclosed, or represents that its use would not infringe privately owned rights. Reference herein to any specific commercial product, process, or service by trade name, trademark, manufacturer, or otherwise does not necessarily constitute or imply its endorsement, recommendation, or favoring by the United States Government or any agency thereof. The views and opinions of authors expressed herein do not necessarily state or reflect those of the United States Government or any agency thereof. 
order to improve statistics, we calculated propagators from 32 different sources per configuration. The 32 sources chosen were those with $x, y$ and $z$ coordinates either 1 or 9 , and $t$ coordinate 1,9 , 17 or 25 . For comparison with other work in the field we give, in Table 1, our estimates for simple properties of S- and P-wave charmonium obtained from these propagators. Using mean field [2] estimates for the renormalization constants, we find that the renormalised quark mass is $M_{R}=0.605$ (lattice units), and the mass of S-wave charmonium is $M_{S}=3.36 \mathrm{GeV}+O\left(v^{4}\right)$, calculated from the $p=0$ state, and $M_{s} d y n=3.00 \mathrm{GeV}+O\left(v^{2}\right)$, calculated from the coefficient of $p^{2}$ in the energy of the $p=2 \pi / 16$ state.

Table 1

Properties of S- and P-wave charmonium from our simulations. $R(r)$ are the radial wave functions. The lattice quantities include mean field renormalizations

\begin{tabular}{|c|c|c|}
\hline & LATTICE & EXPERIMENT \\
\hline$M_{P}-M_{S}$ & $0.40-0.48 \mathrm{GeV}$ & $0.43 \mathrm{GeV}$ \\
$\left|R_{1 s}(0)\right|^{2}$ & $0.599(4) \mathrm{GeV}^{3}$ & $0.48(4) \mathrm{GeV}^{3}$ \\
$\left|R_{1 P}^{\prime}(0)\right|^{2}$ & $0.032-0.053 \mathrm{GeV}^{5}$ & $0.054(13) \mathrm{GeV}^{5}$ \\
\hline
\end{tabular}

The matrix elements we wish to calculate are defined as

$$
\begin{aligned}
& G_{1}=\left\langle{ }^{1} S\left|\psi^{\dagger} \chi \chi^{\dagger} \psi\right|^{1} S\right\rangle / M_{Q}^{2} \\
& H_{1}=\left\langle{ }^{1} P\left|\psi^{\dagger}(i / 2) \vec{D} \chi \chi^{\dagger}(i / 2) \vec{D} \psi\right|^{1} P\right\rangle / M_{Q}^{4} \\
& H_{8}=\left\langle{ }^{1} P\left|\psi^{\dagger} T^{a} \chi \chi^{\dagger} T^{a} \psi\right|^{1} P\right\rangle / M_{Q}^{2},
\end{aligned}
$$

each of which has the generic form

$W=\langle\Phi|O(x, t)| \Phi\rangle$

where $|\Phi\rangle$ is the quarkonium state and $O$ the 4 fermi operator. On the lattice, we measure $W^{*}$ defined by

$W^{*}\left(T, T^{\prime}\right)=\frac{\left\langle 0\left|\phi(-T) O(x, t) \phi^{\dagger}\left(T^{\prime}\right)\right| 0\right\rangle}{\left\langle 0\left|\phi(-T) \phi^{\dagger}(0)\right| 0\right\rangle\left\langle 0\left|\phi(0) \phi^{\dagger}(T)\right| 0\right\rangle}(8)$

where $\phi(t)=\sum_{x} \chi^{\dagger}(x, t) \psi(x, t)$ for the S-wave matrix element and $\phi(t)=\sum_{x} \chi^{\dagger}(x, t) \vec{\Delta} \psi(x, t)$ for the P-wave matrix elements. Then as $T, T^{\prime} \rightarrow$ $\infty$

$$
\begin{aligned}
& G_{1}^{*}\left(T, T^{\prime}\right) \rightarrow G_{1} \frac{2 \pi M_{Q}^{2}}{3\left|R_{1 S}(0)\right|^{2}}=1+\mathcal{O}\left(v^{4}\right) \\
& H_{1}^{*}\left(T, T^{\prime}\right) \rightarrow H_{1} \frac{2 \pi M_{Q}^{4}}{9\left|R_{1 P}^{\prime}(0)\right|^{2}}=1+\mathcal{O}\left(v^{2}\right) \\
& H_{8}^{*}\left(T, T^{\prime}\right) \rightarrow H_{8} \frac{2 \pi M_{Q}^{2}}{9\left|R_{1 P}^{\prime}(0)\right|^{2}}
\end{aligned}
$$

In Fig. 1a we have plotted $G_{i}^{*}$ as a function of $T$, with separate points for each value of $T^{\prime}$, with the restriction $T+T^{\prime}<32$. We notice that $G_{1}^{*}$ is very close to 1 , as expected. In Fig. 1b we have made a similar plot for $H_{1}^{*}$, distinguishing those points with $T<8$ from those with $T \geq 8$. Here, again the values cluster around 1 as expected. Since the statistical errors in these points are comparable with their departure from unity, it is unclear whether the much larger variation for $B_{i}^{*}$ than for $G_{i}^{*}$ is due to the expected $\sim v^{2}$ departure from unity, the increased errors, or both.

Finally, in Fig. 1c we have plotted $H_{8}^{*}$ against $T$. The scatter of points is considerable. The best we can say is that $H_{8}^{*}$ appears to lie in the range $0.1-0.2$, but there is really no good evidence that we have seen a plateau. Experimental data suggest a value [1] around 0.5 (with an error of about $50 \%$ ). It is easy to see why our measurement would be expected to underestimate this quantity. Noise dominates the signal for $T, T^{\prime} \gtrless 8$. However, we know that even at $T=8$ the coefficient of the exponential in each of the propagators in the denominator of (10) has not reached its asymptotic value, and that this value is approached from above. The numerator, on the other hand, is zero at zero separaicion, and hence the coefficient of the exponential in the numerator approachs its asymptotic value from below. Thus, the overall effect of not being able to attain large enough separations is to cause us to underestimate $B_{8}$.

Since this conference, we have extended this analysis to the $b \bar{b}$ system. Here one might hope that, since the radius of the $\Upsilon$ is less than half that of charmonium, the use of a point source would be less of a handicap. Indeed, for $G_{i}^{*}$ and $H_{i}^{*}$ this appears to be the case. Unfortunately, 

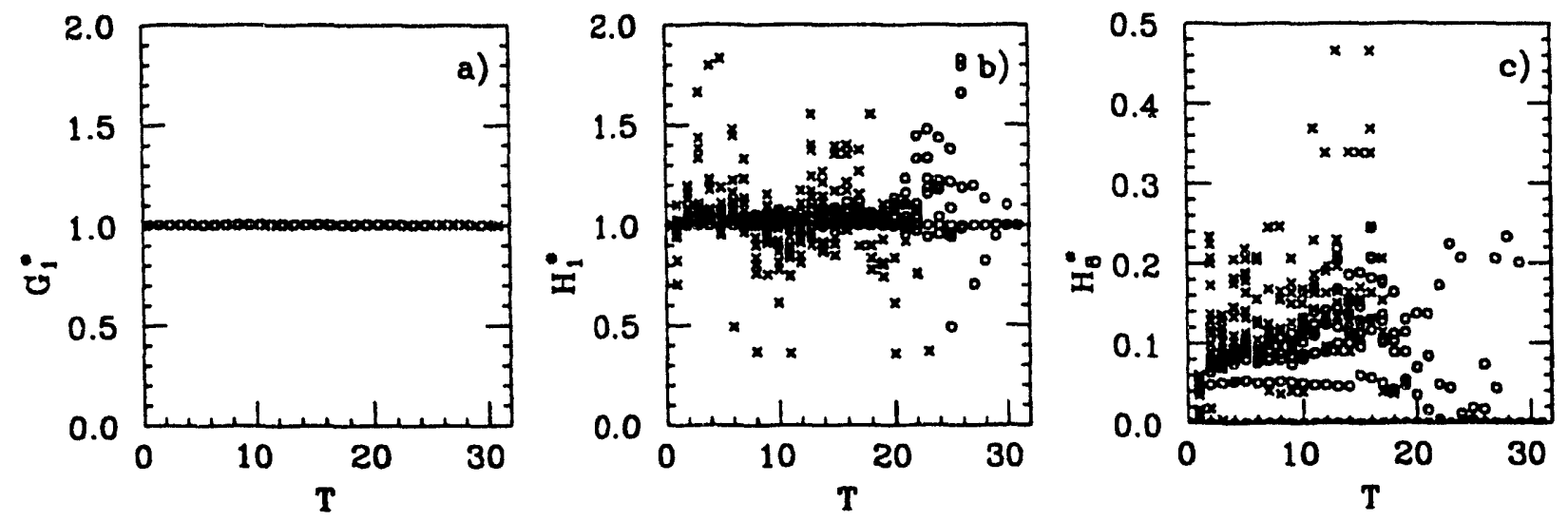

Figure 1. Matrix elements as a function of T. a) $G_{1}^{*}$. b) $H_{1}^{*}$. c) $H_{8}^{*}$. Separate points are plotted for each $T^{\prime \prime}$. Points with $T^{\prime}<T$ are labelled o; those with $T^{\prime} \geq T$ are labelled $x$ in (b) and (c).

for $H_{8}^{*}$ this is not true. $H_{8}^{*} \sim 1 / M_{Q}^{2}$, and so decreases by roughly an order of magnitude in going from the charmonium to the $b \bar{b}$ system. The signal/noise ratio is, if anything, worse for $\chi_{b}$.

In summary we have found that the colour singlet matrix elements involved in the decay of $S$ and P-wave quarkonia $\left(G_{1}\right.$ and $\left.H_{1}\right)$ appear to be related to the quark wave-functions at the origin in the expected manner. To make reliable estimates of the colour-octet matrix element $\left(H_{8}\right)$ contributing to $P$-wave decay would require much higher statistics and, possibly, a longer lattice than we have used. This operator is of considerable interest, since it involves an explicit gluon exchange and cannot, therefore, be estimated in a potential model. At least for the charmonium case, it involves the exchange of gluons which are too soft for one to believe the perturbative estimates.

In the above we have used mean-field estimates of the renormalization constants. For a more thorough analysis, a complete perturbative calculation will be needed. We are now investigating the possibility of improving our signal/noise ratio by using extended sources in a way that is similar to their use in B parameter calculations [3]. Extensions of our calculations to include contributions which are higher order in $v^{2}$ are under consideration.

We wish to thank John Sloan, Christine Davies and G. Peter Lepage for informative discussions and insight. We thank J. B. Kogut, M.-P. Lombardo and D. K. Sinclair for allowing us to use their gauge configurations. These calculations were performed on the CRAY C-90 at NERSC, whose resources were made available to us through the Energy Research Division of the U.S. Department of Energy.

\section{REFERENCES}

1. G. T. Bodwin, E. Braaten and G. P. Lepage, Phys. Rev. D46, R1914, (1992); G. T. Bodwin, E. Braaten and G. P. Lepage, in "The Fermilab Meeting DPF'92n, 1063, C. H. Albright, P. H. Kasper, R. Raja and J. Yoh (eds.), World Scientific, Singapore, (1993); G. T. Bodwin, E. Braaten, T. C. Yuan and G. P. Lepage, Phys. Rev D46, R3703, (1992).

2. G. P. Lepage, L. Magnea, C. Nakhleh, U. Magnea and K. Hornbostel, Phys. Rev. D46, 4052, (1992); B. A. Thacker and G. P. Lepage, Phys. Rev. D43, 196 (1991); J. Sloan, poster presented at LATTICE'93, Dallas, Texas (1993).

3. R. Gupta, D. Daniel, G. W. Kilcup, A. Patel and S. R. Sharpe, Phys. Rev. D47, 5113, (1993). 

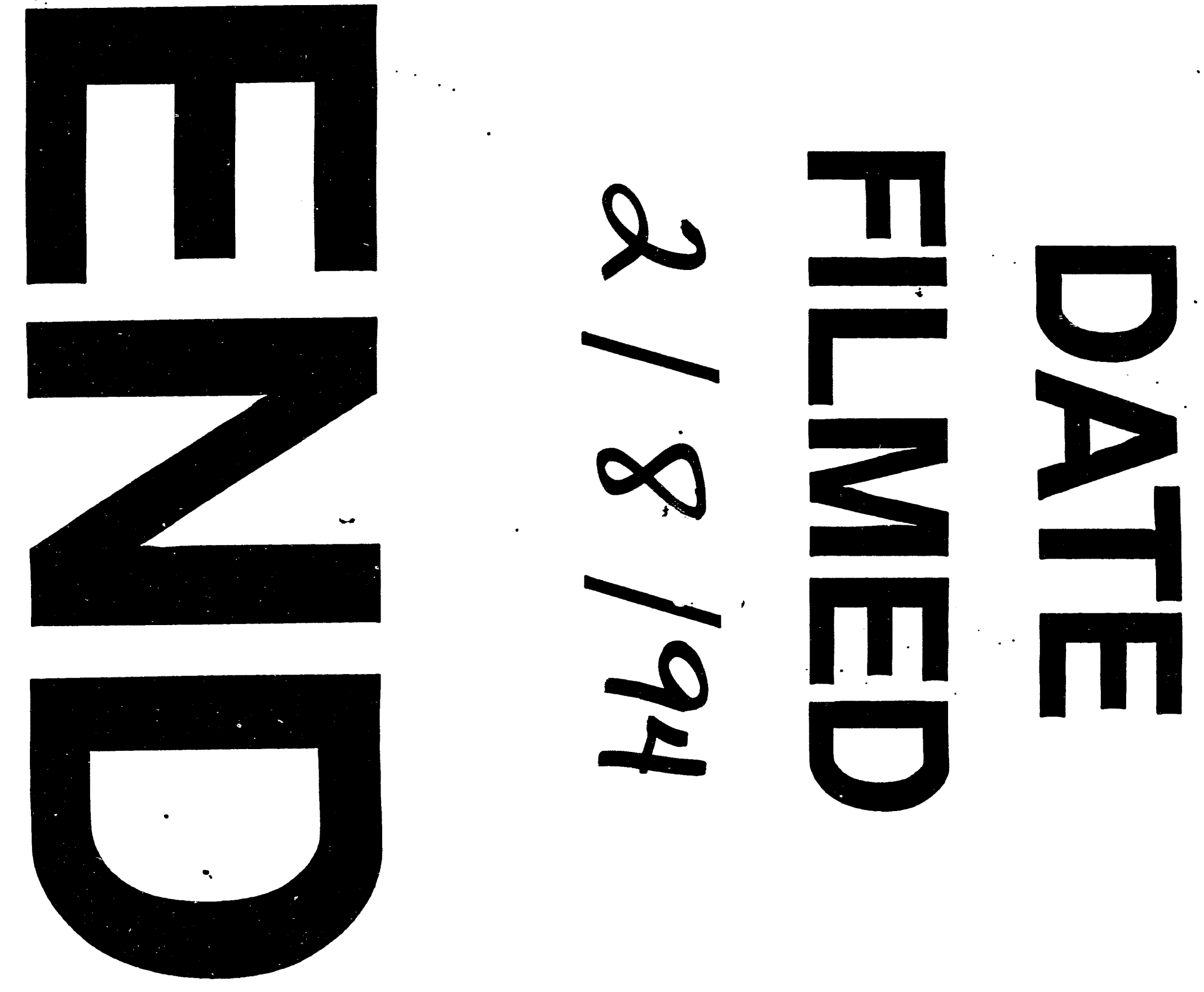


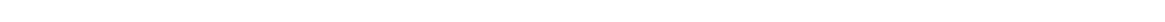

